\title{
Publisher's Note: Towards the Solution of the Many-Electron Problem in Real Materials: Equation of State of the Hydrogen Chain with State-of-the-Art Many-Body Methods [Phys. Rev. X 7, 031059 (2017)]
}

Mario Motta, David M. Ceperley, Garnet Kin-Lic Chan, John A. Gomez, Emanuel Gull, Sheng Guo, Carlos A. Jiménez-Hoyos, Tran Nguyen Lan, Jia Li, Fengjie Ma, Andrew J. Millis, Nikolay V. Prokof'ev, Ushnish Ray, Gustavo E. Scuseria, Sandro Sorella, Edwin M. Stoudenmire, Qiming Sun, Igor S. Tupitsyn, Steven R. White, Dominika Zgid, and Shiwei Zhang

(Received 18 March 2021; published 6 April 2021)

DOI: 10.1103/PhysRevX.11.029901

This paper was published online on 28 September 2017 with a typographical error in a Grant number in the Acknowledgments. In the Acknowledgments, the third to last sentence should read as "S. R. W. and E. M. S. acknowledge support from the U.S. Department of Energy, Office of Science, Basic Energy Sciences under Grant No. DE-SC0008696." The Acknowledgments have been corrected as of 24 March 2021. The Acknowledgments are incorrect in the printed version of the journal.

Published by the American Physical Society under the terms of the Creative Commons Attribution 4.0 International license. Further distribution of this work must maintain attribution to the author(s) and the published articles title, journal citation, and DOI. 